Classification

Physics Abstracts

$75.10 \mathrm{H}-75.60 \mathrm{C}-75.70$

\title{
Properties of the branched state of an Ising dipolar magnet
}

\author{
M. Gabay and T. Garel \\ Laboratoire de Physique des Solides (*), Université Paris-Sud, Centre d'Orsay, \\ 91405 Orsay Cedex, France
}

(Reçu le 22 juin 1984, accepté le 29 août 1984)

\begin{abstract}
Résumé. - On considère les propriétés thermodynamiques de l'état branché d'un ferromagnétique dipolaire de type Ising confiné dans une plaque d'épaisseur $D$. Les modèles introduits par Lifshitz et Privorotskii nous permettent (i) de dériver, à la limite des grandes épaisseurs $D$, des lois d'échelle pour la période de la structure, l'aimantation de surface et le nombre de branchements, en l'absence de champ magnétique $H$, dans toute la gamme de températures $0 \leqslant T \leqslant T_{0}\left(T_{0}\right.$ est la température de Curie du système branché); (ii) de prédire, en utilisant les résultats numériques concernant la configuration d'équilibre de l'état à un branchement en présence de champ, qu'augmenter $H$ à $D$ et $T$ fixés induit, dans l'état multiplement branché, une série de transitions du premier ordre vers des états de moins en moins ramifiés. L'allure des courbes critiques $D_{\mathrm{C}}(H)$ est donnée; (iii) de montrer que la surface de l'état quasi-infiniment branché possède une susceptibilité constante pour toute température $T \leqslant T_{0}$.
\end{abstract}

\begin{abstract}
We consider the thermodynamic properties of the branched state of an Ising dipolar magnet confined in a slab of thickness $D$. Within the framework of the Lifshitz and Privorotskii models (i) we derive, for large $D$, scaling laws for the period of the structure, the surface magnetization, and the number of branching, for zero external field $H$ in the whole range $0 \leqslant T \leqslant T_{0}\left(T_{0}\right.$ is the Curie temperature of the branched structure); (ii) Using the results of a numerical study on the equilibrium configuration of the singly-branched system when $H$ is present, we predict that increasing $H$ at fixed $D, T$ causes the multiply-branched system to undergo a series of first order transitions to lesser and lesser ramified states. A phase diagram for the critical curve $D_{\mathrm{C}}(H)$ is given; (iii) The surface state of the quasi-infinitely branched state is shown to possess a constant susceptibility for all $T \leqslant T_{0}$.
\end{abstract}

\section{Introduction.}

Dipolar forces in ferromagnets play a central role in determining the equilibrium spatial configurations of a finite size specimen : indeed, these forces induce magnetic poles on the surface of the sample thus increasing the magnetostatic energy; to reduce it the system breaks into domains the shape and size of which are determined by a balance between exchange, anisotropy and magnetostatic energies. From a theoretical point of view it is easier to consider the case of an Ising magnet confined in a slab of thickness $D$, with its magnetization normal to the slab (Figs. 1, 2). For $D$ not too large, the equilibrium state consists in a stripe structure (Fig. 1) where up and down domains alternate regularly [1]. However, there exists a critical value $D_{\mathrm{C}}$ of $D$

(*) Laboratoire associé au C.N.R.S. 


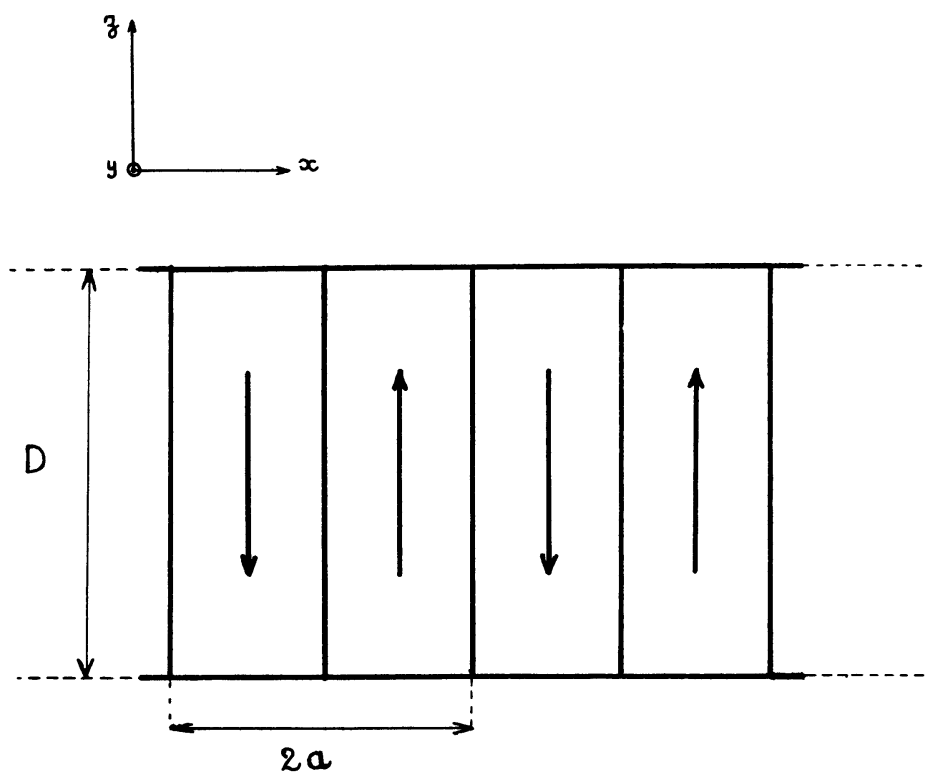

Fig. 1. - Stripe structure in zero field for an Ising dipolar magnet in a slab of thickness $D$; the period is $2 a$.

beyond which the previous state becomes unstable and ramifications develop from the surface : this is the so-called branched state where domains of the "down " state nucleate in domains of the " up " state and vice versa (Fig. 2) [2]. As $D$ increases further, the system becomes more and more ramified in a more or less self-similar fashion. Again let us stress that this process mostly affects the surface of the sample in order to effectively reduce the magnetostatic energy. Various models describing the branched state have been constructed, using simplifying assumptions $[2,3]$. So far their predictions pertain to the zero temperature $(T=0)$ zero external field $(H=0)$ situation. It is the purpose of the present Letter to exhibit simple laws for these structures, valid in the whole range $0 \leqslant T \leqslant T_{0}$ ( $T_{0}$ is the Curie temperature of the branched state) and in the presence of $H$. These concern $a$, the half periodicity of the branched state, $n_{\mathrm{S}}$ the equilibrium number of ramifications on the surface, $m_{\mathrm{S}}$ the surface magnetization (the number of up spins minus the number of down spins on the surface, times $M$ the bulk magnetization) and $D_{\mathrm{C}}(H)$ the first order lines separating the stability regions of the various branched phases in the $D, T$ plane.

We show that for $H=0$ and large $D$, when $T$ increases from 0 to $T_{0}$,

(i) $a$ decreases :

$$
a \underset{T \rightarrow 0}{\sim} \delta^{1 / 3} D^{2 / 3} \text { whereas } a \underset{T \rightarrow T_{0}}{\sim} \delta^{1 / 2} D^{1 / 2}
$$

(ii) $n_{\mathrm{S}}$ increases slightly :

$$
n_{\mathrm{S}} \underset{T \rightarrow 0}{\sim} \frac{2}{3} \ln \left(\frac{D}{\delta}\right) \text { whereas } n_{\mathrm{S}} \underset{T \rightarrow T_{0}}{\sim} \ln \left(\frac{D}{\delta}\right)
$$

(iii) $m_{\mathrm{S}} / M$ decreases :

$$
m_{\mathrm{S}} / M \underset{T \rightarrow 0}{\sim}\left(\frac{\delta}{D}\right)^{2 / 3} \text { whereas } m_{\mathrm{S}} / M \underset{T \rightarrow T_{0}}{\sim} \frac{\delta}{D}
$$

$\delta$ is the zero temperature wall thickness and $M$ the equilibrium bulk magnetization at a given $T$. 


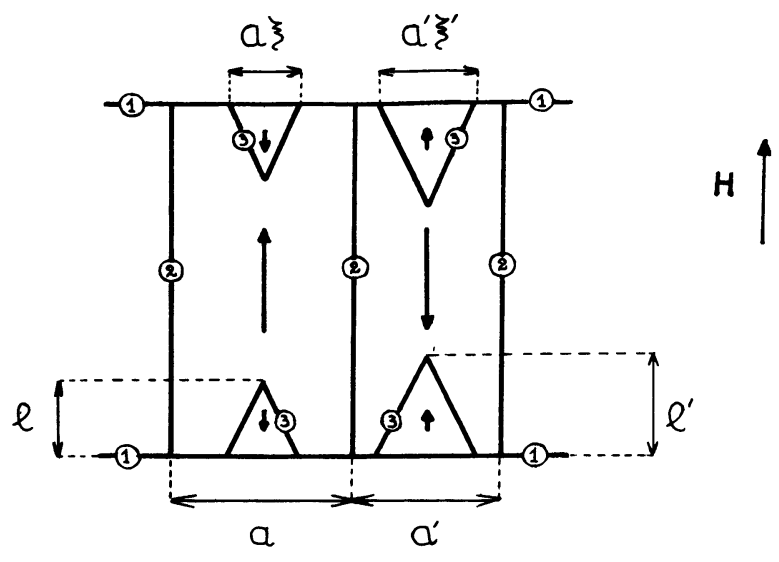

(a)

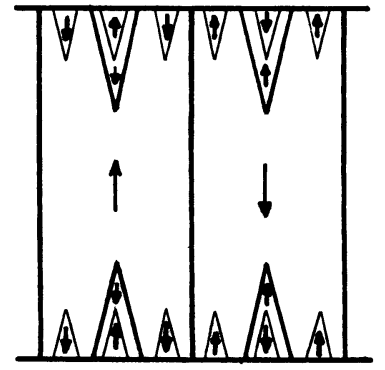

(b)

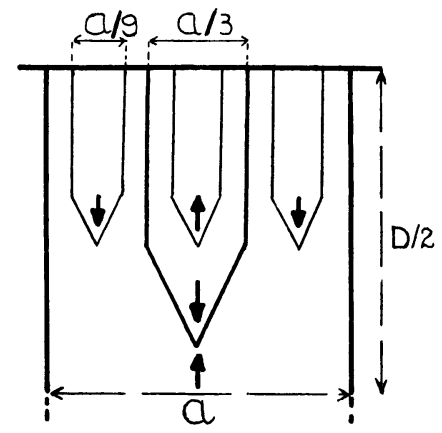

(C)

Fig. 2. - (a) Singly branched structure in a field $H$ in the Lifshitz theory. The underlying stripe structure yields contributions $E_{\mathrm{W}}$ to the free energy in regions 2 and $E_{\mathrm{DS}}$ in regions 1 (boundaries of the sample) over the period $a+a^{\prime}$. The schematically drawn branched parts give a contribution $E_{\mathrm{B}}$ in regions 3 consisting of a wall part and a magnetostatic part due to poles on the boundaries between up and down domains. When $H=0, a=a^{\prime}, l=l^{\prime}, \xi=\xi^{\prime}$. (b) The $H=0$ doubly branched state in the Lifshitz model for a period of the structure. (c) The $H=0$ doubly branched state in the Privorotskii model for a half period.

Within the framework of the Lifshitz [2a] model we have obtained a variational free energy describing the behaviour of the singly branched state in a field $H$. A numerical solution shows that

(i) for low fields the branched structure is stable against distortions;

(ii) there exists a critical field $H_{\mathrm{C}}$ where the system jumps into the stripe phase ;

(iii) analytically continuing the metastable branched solution for $H>H_{C}$ shows an evolution to a stripe phase as well. Simple physical considerations show that when $N$ stages of branching are present, increasing $H$ at fixed $D$ and $T$ causes the system to undergo a series of first order transitions to lesser and lesser ramified states. The critical field $H_{\mathrm{C}}$ corresponding to the transition from the $N$ th to the $(N-1)$ th structure (that is to the first transition occurring when $H$ 
is increased) is such that, for large $D$,

$$
H_{\mathrm{C}} / M \underset{T \rightarrow 0}{\sim}\left(\frac{\delta}{D}\right)^{2 / 3} \text { whereas } H_{\mathrm{C}} / M_{T \rightarrow T_{0}}^{\sim} \frac{\delta}{D} .
$$

Using equation (3) shows that

$$
m_{\mathrm{S}} \sim H_{\mathrm{C}} \text { for all } T
$$

indicating that the surface state of the system has a constant susceptibility; the infinitely branched system thus displays a similar type of behaviour as is observed in the bulk of the SK model of an Ising spin glass [4], for all $T$ below the transition temperature.

\section{The zero field case.}

2.1 The LOW TeMPeraxure Regime. - According to Lifshitz [2a], the $H=T=0$ excess free energy per unit area of a singly branched structure takes on the following form

$$
F=E_{\mathrm{w}}+E_{\mathrm{B}} / 2 a+E_{\mathrm{Ds}} .
$$

A quantitative discussion of each term in equation (6) is postponed to an accompanying longer article [5]; yet figure 2a shows their meaning :

$$
E_{\mathrm{w}}=\sigma_{\mathrm{w}} D / a
$$

is the wall energy per unit area of the underlying bulk stripe structure (that is of the vertical walls). $\sigma_{\mathrm{w}}$ is the surface tension of the walls and is related, at $T=0$, to the characteristic wall half width $\delta$ by $\delta=\sigma_{\mathrm{w}} / M^{2}$.

$E_{\mathrm{B}}$ characterizes the increase in $F$ brought about when branching occurs. This increase has two sources, namely an extra contribution to the wall energy due to the ramification and a volume demagnetizing energy due to magnetic poles localized on the surface of the ramification. We only quote from Lifshitz

$$
E_{\mathrm{B}}=8 M^{2} \delta l+\frac{32}{9} \pi M^{2} \frac{a^{3}}{l}\left[1-(1-\xi)^{3 / 2}\right]^{2}
$$

where the ramification extends to a depth $l$ from the surface and has a width $a \xi$ on the surface.

$E_{\mathrm{DS}}$ is the demagnetizing energy per unit area due to poles on the boundaries of the slab. Its exact form, given in reference [5], can be reasonably estimated to behave like

$$
E_{\mathrm{DS}} \approx \gamma a_{\mathrm{s}} M^{2}
$$

$\gamma$ is a numerical constant of order one and $a_{\mathbf{S}}$ is the periodicity on the surface. This form is not unexpected since the demagnetizing energy is obtained from the pole distribution on the surface. Branching causes the surface periodicity to assume a much lower value than the bulk's, thus considerably reducing $E_{\mathrm{Ds}}$. If $D$ is large enough, this decrease largely compensates the additional term $E_{\mathrm{B}}$, favouring the branched structure. The equilibrium values of $a, l, \xi$ are obtained by varying $F$ with respect to these parameters (in particular, since $l$ only appears in $E_{\mathrm{B}}$, it is possible to get $l(a, \xi)$ by expressing the condition $\left.\partial E_{\mathrm{B}} / \partial l=0\right)$. Yet, when $N$ generations of branching are present, $F$ depends on $2 N+1$ parameters, so it is not possible to extend Lifshitz's results in a simple way. We use instead Privorotskii's scheme [3], illustrated in figure $2 \mathrm{c}$ :

(i) the surface (i.e. maximum) width of the first generation is $a / 3$; that of the second generation is $1 / 3$ that of the previous generation, i.e. $a / 9$, and so on;

(ii) each ramification spike is surrounded by two neighbouring vertical walls. 
Since vertical walls carry no magnetic surface charge, the contribution of each spike to the free energy, at any level $n, 0 \leqslant n \leqslant N$, is given by an $E_{\mathrm{B}}^{(n)}$ equivalent to equation (7b) with $\xi=1 / 3$ and where $a$ is replaced by $a / 3^{n-1}$. The corresponding $l_{n}$ is found again with the condition $\partial E_{\mathrm{B}}^{(n)} / \partial l_{n}=0$, yielding

$$
\begin{aligned}
l_{n} & =\frac{2}{3} \sqrt{\frac{\pi}{\delta}} \frac{a^{3 / 2}}{3^{3(n-1) / 2}}\left[1-\left(\frac{2}{3}\right)^{3 / 2}\right] \\
E_{\mathrm{B}}^{(n)} & =\frac{8}{3} M^{2} \sqrt{\pi \delta} \frac{a^{3 / 2}}{3^{3(n-1) / 2}}\left[1-\left(\frac{2}{3}\right)^{3 / 2}\right]
\end{aligned}
$$

the excess energy due to each spike and its neighbouring walls (shared with another spike) is

$$
E_{\mathrm{B}}^{(n)}+M^{2} \delta l_{n}=\frac{10}{3} M^{2} \sqrt{\pi \delta} \frac{a^{3 / 2}}{3^{3(n-1) / 2}}\left[1-\left(\frac{2}{3}\right)^{3 / 2}\right]
$$

For an $N$ branched structure, we obtain,

$$
4 \sum_{n=1}^{N} 3^{n-1}\left(E_{\mathrm{B}}^{(n)}+M^{2} \delta l_{n}\right)=\frac{40}{3} M^{2} \sqrt{\pi \delta a^{3}}\left[1-\left(\frac{2}{3}\right)^{3 / 2}\right] \frac{1-3^{-N / 2}}{1-3^{-1 / 2}}
$$

Similarly the wall energy of the underlying bulk stripe $\cdot$ structure is given by

$$
2 \sigma_{\mathrm{w}}(D-2 L) \text { where } L=\sum_{n=1}^{N} l_{n}
$$

The $4 \sigma_{\mathrm{w}} L$ term avoids double counting of the wall energy included in equations (8c-8d).

The free energy per unit area $F$ reads

$$
F=\lambda M^{2} \delta^{1 / 2} a^{1 / 2}\left(\frac{4}{5}-X+\frac{X^{3}}{5}\right)+M^{2} \delta \frac{D}{a}+E_{\mathrm{DS}}
$$

where

$$
X=3^{-N / 2} \text { and } \lambda=\frac{20}{3} \sqrt{\pi} \frac{\left[1-\left(\frac{2}{3}\right)^{3 / 2}\right]}{1-3^{-1 / 2}}
$$

In Privorotskii's scheme it is easy to calculate $E_{\mathrm{DS}}$ exactly. One finds (see also Eq. (12) of Ref. [5])

$$
E_{\mathrm{DS}}=\frac{16}{\pi^{2}} M^{2} X^{2} a \sum_{(n \text { odd })} \frac{1}{n^{3}}\left(1-\exp \left(-n \pi D /\left[a X^{2}\right]\right)\right)
$$

For large $N$, the exponential term can be omitted, yielding

$$
E_{\mathrm{DS}}=\gamma M^{2} a X^{2}, \quad \gamma=1.71 .
$$

Varying $F$ with respect to $X$ and $a$ gives

$$
\begin{gathered}
\left(\frac{a}{\delta}\right)^{1 / 2}=\frac{\lambda}{2 \gamma} \frac{1}{X}\left(1-\frac{3}{5} X^{2}\right) \\
\frac{\lambda}{2}\left(\frac{4}{5}-X+\frac{X^{3}}{5}\right)\left(\frac{\delta}{a}\right)^{1 / 2}-\frac{\delta D}{a^{2}}+\gamma X^{2}=0
\end{gathered}
$$


leading to

$$
\frac{\lambda^{4}}{16 \gamma^{3}} \frac{1}{X^{3}}\left(1-\frac{3}{5} X^{2}\right)^{3}\left(\frac{4}{5}-\frac{2}{5} X^{3}\right)=\frac{D}{\delta} .
$$

Equations (10a) and (11) determine $a / \delta$ and $X$ as a function of $D / \delta$. Since this model is, by construction, only meaningful for integer values of $N$, the critical thickness for the first $1 / 3$ branching structure in this model is obtained for $X=\frac{1}{3^{1 / 2}}$ :

$$
\frac{D_{\mathrm{C}}^{(1)}}{\delta} \simeq 633.29
$$

Critical thresholds for successive generations of branching are given by equation (11); they can be written as

$$
D_{\mathrm{C}}^{(N)} \sim f(X) \delta
$$

where $f(X)$ is obtained from equation (11) and where $X$ is replaced by $1 / 3$ for the 2 nd generation, $1 / 3^{3 / 2}$ for the $3 \mathrm{rd}$, and so on.

For a given $D$, equation (11) yields

$$
X^{-3} \sim \frac{D}{\delta} \text { for } D \text { large }
$$

whence

$$
n_{\mathrm{S}} \underset{D \rightarrow \infty}{\sim} \frac{2}{3} \ln \left(\frac{D}{\delta}\right)
$$

and

$$
a \sim \delta^{1 / 3} D^{2 / 3}
$$

in agreement with previous results.

Furthermore, given that in this model $m_{\mathrm{S}} / M= \pm 1 / 3^{N}$

and

$$
m_{\mathrm{S}} / \underset{T \rightarrow 0}{\sim}\left(\frac{\delta}{D}\right)^{2 / 3}
$$

$$
F \underset{T \rightarrow 0}{\sim} M^{2} a^{1 / 2} \delta^{1 / 2} \underset{T \rightarrow 0}{\sim} M^{2}\left(\delta^{2} D\right)^{1 / 3}
$$

2.2 INCREASING $T$. - As $T$ grows, the surface tension $\sigma_{\mathrm{w}}$ decreases in magnitude. Typically, in a Ginzburg-Landau theory $\sigma_{\mathrm{W}} \underset{T \rightarrow T_{0}}{\sim} \delta^{2} M^{2} \xi_{\mathrm{C}}^{-1}$ where $\xi_{\mathrm{C}}$ is the coherence length of the system. It varies from $\delta$ at $T=0$ to $\left[\left(T_{0}-T\right) / T_{0}\right]^{-1 / 2} \delta$, close to the critical point, and sets the scale of the characteristic wall width. When $\xi_{\mathrm{C}} \sim a$, the non-interacting wall theory that we have used for $T=0$ breaks down. Fluctuations become important and the system crosses over to the critical regime. However, for dipolar interactions the size of that critical region is not too large ; therefore the behaviour of $a, D_{\mathrm{C}}^{(N)}, n_{\mathrm{S}}$ and $m_{\mathrm{S}}$ in the critical regime can be fairly accurately extrapolated from the crossover region (see also Ref. [6]). Also, since the upper critical dimensionality for this problem is $d_{\mathrm{C}}=3$, exponents deduced from this extrapolation should be correct to leading order. The extrapolation procedure goes as follows : we first replace $\delta$ by the actual value $\sigma_{\mathrm{w}} / M^{2}$ in equations (7b-13d); using the fact that $\sigma_{\mathrm{w}} / M^{2} \sim \delta^{2} a^{-1}$ in the crossover region, we conclude from (12) to (13d) that for $T \rightarrow T_{0}$ and $D$ large,

$$
D_{\mathrm{C}}^{(N)} \underset{T \rightarrow T_{0}}{\sim} \delta^{3 / 2} D^{-1 / 2} f(X)
$$




$$
\begin{aligned}
& a \underset{T \rightarrow T_{0}}{\sim} \delta^{1 / 2} D^{1 / 2} \\
& n_{\mathrm{S}} \underset{T \rightarrow T_{0}}{\sim} \ln \left(\frac{D}{\delta}\right) \\
& m_{\mathrm{S}} / \boldsymbol{M}_{T \rightarrow T_{0}}^{\sim} \frac{\delta}{D} .
\end{aligned}
$$

Two comments are in order.

(i) $D_{\mathrm{C}}^{(N)}, a, n_{\mathrm{S}}$ are finite quantities when $T \rightarrow T_{0}$, as long as $D$ remains finite. Also, as can be seen from equations (12) and $(14 \mathrm{a}) D_{\mathrm{C}}^{(N)}\left(T \lesssim T_{0}\right) \ll D_{\mathrm{C}}^{(N)}(0)$ for large $D$ fixed. Increasing $T$ thus favours branching. Starting from a, say, stripe configuration at $T=0$, it is possible to generate a branched structure at higher temperatures provided that $D \lesssim D_{\mathrm{C}}^{(1)}(0)$. Similarly, higher generations of branching can be obtained at finite temperatures starting from a branched state at $T=0$. This property is also contained in equations (13a) and (14c) which show that for $D$ large fixed, $n_{\mathrm{S}}\left(T \lesssim T_{0}\right)>n_{\mathrm{S}}(0)$.

(ii) A more rigorous way of deriving equation (14) would be to construct a Ginzburg-Landau free energy functional $F(m(x, z))$ for $T \simeq T_{0}$. In the branched case, the magnetization density $m(x, z)$ is a space dependent quantity in both $x$ and $z$. Just as in the stripe case [6,7], the $x$ dependence of $m(x, z)$ may be described by a $\cos \left(q_{x} x\right)$ term with $q_{x} \sim \frac{\pi}{a}$. To represent the $z$ dependence is trickier since, at $T=0, m(x, z)$ already has a complicated structure and varies abruptly as $z$ goes from $-D / 2$ to $+D / 2$. Still it is possible to define a coarse grained magnetization density

$$
\bar{m}(z)=\frac{1}{a \Delta z} \int_{-a / 2}^{+a / 2} \int_{z}^{z+\Delta z} \mathrm{~d} x^{\prime} \mathrm{d} z^{\prime} m\left(x^{\prime}, z^{\prime}\right)
$$

where $\Delta z$ is a small arbitrary thickness; $\bar{m}(z)$ decreases smoothly when $z$ varies from 0 to $\pm D / 2$. It is by definition an even function of $z$. Only for $D \rightarrow \infty$ do we have $\bar{m}( \pm D / 2)=0$. At $T=0$ the full magnetization density profile can thus be approximated by a coarse grained form $\varepsilon(x) \bar{m}(z)$; $\varepsilon(3 i)$ is a periodic function of $x$ of period $2 a: \varepsilon(x)=+1$ for $x \in(-a, 0), \varepsilon(x)=-1$ for $x \in(0, a)$. Close to $T_{0}$, we may, therefore, take $m(x, z) \sim \bar{m}(z) \cos \left(q_{x} x\right)$. For $D \rightarrow \infty$ a Fourier analysis yields $m(x, z) \simeq M \cos \left(q_{z} z\right) \cos \left(q_{x} x\right)$ with $q_{z} \sim \pi / D$. For finite $D$, one clearly has more $q_{z}$ modes present (e.g. $q_{z}=0$, since $\bar{m}( \pm D / 2) \neq 0$ ). This type of approach has been considered by Barker and Gehring (private communication).

\section{Turning a magnetic field on.}

In the presence of an external field $H$, the equilibrium configuration of the system will be distorted. As we report below, the magnitude of $H$ for which these distortions become relevant is much larger than that where first-order transitions occur. With that in mind let us rewrite the free energy $F$ in the presence of the Zeeman term. For small $H$,

$$
F(H) \simeq F(0)-\frac{H}{2 a} \int_{-a}^{+a} \int_{-D / 2}^{+D / 2} \mathrm{~d} x \mathrm{~d} z m(x, z) .
$$

A natural rescaling for this problem is achieved by redefining $F, x, z$ and $m$, namely : $x / 2 a=\tilde{x}$, $z / l=\tilde{z}, m / M=\tilde{m}$ and $F / M^{2} \delta^{1 / 2} a^{1 / 2}=\tilde{f} ;$ thus

$$
F(H) \simeq M^{2} \delta^{1 / 2} a^{1 / 2}\left(\tilde{f}(0)-\tilde{H} \iint \mathrm{d} \tilde{x} \mathrm{~d} \tilde{z} \tilde{m}(\tilde{x}, \tilde{z})\right)
$$


Introducing dimensionless quantities has allowed us to find the natural scale for the magnetic field $M \delta / a$. In other words, phase transitions occurring for small $H$ values will correspond to fields proportional to $M \delta / a$.

Using the Lifshitz model, we have explicitly considered the effect of $H$ on the singly branched structure. This implies writing a free energy depending upon six variational parameters (Fig. 2a). Extensive details are given in reference [5]. A numerical solution of the variational problem shows that, at $T=0$,

(i) the branched structure is stable against distortions for fields up to $H_{\mathrm{C}} \sim \xi^{\prime} M \delta / a^{\prime}$;

(ii) a first-order transition to the stripe state takes place at $H_{\mathrm{C}}$;

(iii) up to $H_{\mathrm{C}}$, the sample's magnetization and the free energy $F$ almost retain their $H=0$ values ;

(iv) if the metastable branched solution is analytically continued beyond $H_{\mathrm{C}}$, an evolution to the stripe state takes place for $H_{\mathrm{S}} \gg H_{\mathrm{C}}$.

For the multiply branched structure, the Lifshitz model becomes untractable. A simple assumption consists in transposing the evolution of the singly branched state to the $N$ branched case. Physically plausible arguments are presented in reference [5]; an interesting prediction resulting from this assumed behaviour is shown in figure $3:$ it tells that, for low $T$, the number of branchings will decrease in jumps as $H$ is increased at fixed $D, T$. Since $M \delta / a$ sets the scale of $H$, these jumps will occur for fields $H_{\mathrm{C}}^{(N)}$ such that

$$
H_{\mathrm{C}}^{(N)} \sim \frac{M \delta}{a}
$$

For large $D$, i.e. for very ramified structures,

$$
H_{\mathrm{C}}^{(N)} \underset{T \rightarrow 0}{\sim} M\left(\frac{\delta}{D}\right)^{2 / 3} .
$$

The $T \rightarrow T_{0}$ limit can be obtained in a similar fashion, by applying the procedure described in section 2.2. It is reasonable to believe that for $T \rightarrow T_{0}$ these fields also correspond to first-order

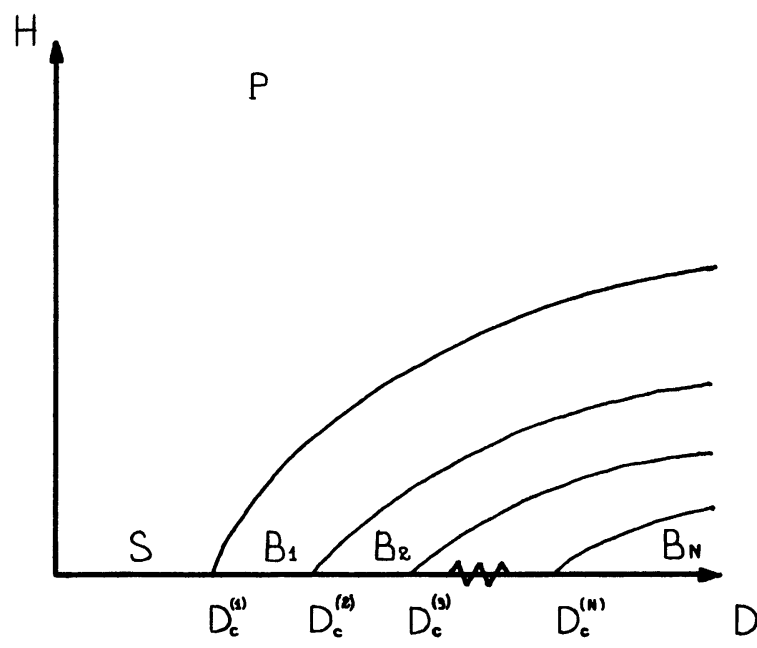

Fig. 3. $-H$ versus $D$ phase diagram showing stability regions of the stripe $(S)$, singly $\left(B_{1}\right)$, doubly $\left(B_{2}\right), \ldots$, $N$ ly $\left(B_{N}\right)$ branched states separated by 1 st order lines $D_{C}^{(N)}(H)$. The boundary with the paramagnetic state $(\mathrm{P})$ is not shown. 
transitions. One then has

$$
H_{\mathrm{C}}^{(N)} \underset{T \rightarrow T_{0}}{\sim} M \frac{\delta}{D} \text { for } D \rightarrow \infty
$$

In the limit of vanishing surface magnetization ( $D$ large) we therefore deduce

$$
m_{\mathrm{S}} \sim H_{\mathrm{C}} \text { for all } T \text { below } T_{0} .
$$

This justifies our statement in the introduction.

\section{Conclusion.}

The branched state of Ising dipolar magnets presents a rich variety of behaviour as a function of temperature and field. Some of our predictions have not yet been tested experimentally. We therefore hope that our study can stimulate experimental work, given the cross-disciplinary implications of it.

\section{Acknowledgments.}

We wish to thank G. Gehring and J. Lajzerovicz for discussions.

\section{References}

[1] Kittel, C., Rev. Mod. Phys. 21 (1949) 541.

Kooy, J., Enz, U., Philips Res. Rep. 15 (1960) 7.

[2] (a) LifSHITZ, E. M., J. Phys. USSR 8 (1944) 337.

(b) KaCZer, J., Sov. Phys. JETP 19 (1964) 1204.

(c) Hubert, A., Phys. Status Solidi 24 (1967) 669.

[3] Privorotskil, I., Thermodynamic theory of domain structures (John Wiley, New York) 1976.

[4] Parisi, G., Philos. Mag. B 41 (1980) 677.

[5] Gabay, M., Garel, T., submitted to J. Physique.

[6] Barker, W. A., Gehring, G. A., J. Phys. C 16 (1983) 6415.

[7] Garel, T., Doniach, S., Phys. Rev. B 26 (1982) 325. 